\title{
Creative Science - injecting innovation into the IT Industry
}

\author{
"The real source of wealth and capital in this new era is not material things, it is the human mind, the \\ human spirit, the human imagination, and our faith in the future" Steve Forbes.
}

Vic Callaghan - The University of Essex (vic@essex.ac.uk)

Product innovation can be both an opportunity and a concern for IT companies. Those driving innovation can gain unprecedented market openings, while those trailing can risk disaster. Companies such as Kodak and Nokia clearly show how disruptive innovations can threaten even the largest technological giants. Hence, companies both small and large have a keen interest in methods with the potential of improving their product innovation capability. In this article, I will describe a new product innovation tool called Science-Fiction Prototyping (SFP) ${ }^{1}$ which, perhaps surprisingly, seeks to encourage scientists and engineers to write fictional stories about their work.

The story of SFP began with Brian Johnson, Intel's Futurist. Intel is the dominant company in the manufacture of integrated circuits (chips) for computers. Companies producing integrated circuits face a particularly difficult challenge as it can take 7+years from the conception of a new chip to its delivery to customers which, in terms of current product and market dynamics, is a very long time. For example, new versions of mobile phones, pads and desktops appear as frequently as every 18 months meaning that chip designers need to think up to 4 generations ahead, a daunting challenge. Compounding the difficulties, traditional engineering education doesn't always prepare engineers well for tasks that need creative skills. Step in Brian David Johnson, Intel's futurist who had the idea that, if engineers were asked to write fiction rather than fact about extrapolating their technologies forward, it might help them escape the shackles of worrying about whether the technology would work, and allow them to make imaginative leaps that, in turn, could serve as motivational visons for their R\&D programmes. The fictions he advocated were based on science, but extrapolated into the future and acting as prototypes to evaluate new ideas ..... Science-Fiction Prototyping (SFP) was born! The deliberately detailed analogy to real-life was an important feature which allowed SFP to function as a prototype (a narrative based simulation of a future reality) whereby different stakeholders could assess the idea's feasibility and usefulness, which differenced it from earlier scenario or design-fiction based approaches.

In parallel, Essex University were faced with the need to make a presentation to a non-technical audience at the 2004 United Nations Habitat Forum, explaining the risks that pervasive computing technologies presented to individual privacy. For that event we adopted a series of fictional vignettes to provide a conversation bridge between the researchers and the largely non-technical audience. The final piece of the jigsaw was a chance meeting with Brian Johnson at IE'07 in Ulm which brought together these two facets of science-fiction, prototyping and communications, giving birth to the Creative Science Foundation CSf (www.creative-science.org) with a mission to facilitate conversations between professionals and society at large, connecting companies/researchers to their customers and thereby enabling conversations about the kind of futures and innovations we all want. In the remainder of this article some current SFP activities will be described.

\footnotetext{
${ }^{1}$ http://www.morganclaypool.com/doi/abs/10.2200/S00336ED1V01Y201102CSL003
} 


\section{The Education Desk}

The first Creative Science Foundation workshop took place in Kuala Lumpur in 2010 and contained an SFP entitled 'Tales from a Pod" ${ }^{2}$, which explored how mixed-reality, nano-technology and Al might combine to create new kinds of education experiences. At the heart of the story was a product innovation called an ePod (educational pod). A short extract from the SFP is as follows:

ePods were effectively small cocoons; something like a comfortable armchair enclosed within a sound-proof egg-like structure packed with sophisticated but largely invisible technology that included immersive mixed reality and sophisticated Al. The immersive reality technology aimed to make the participant feel as though they were truly part of a fictional physical world.

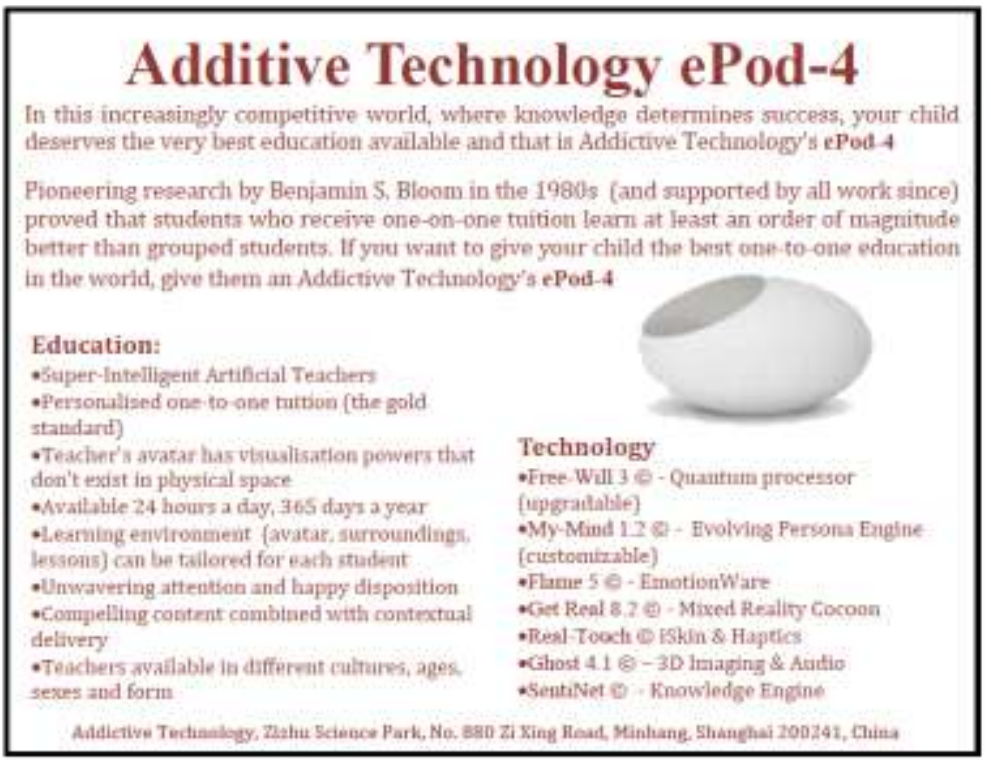

Figure 1: A fake sales brochure from the 'Tales from a pod' SFP

A fake product advertisement from the SFP is shown in Figure 1, illustrating the fidelity they seek to achieve. In addition to the story, SFPs can include supporting material such as product specifications, marketing material etc.
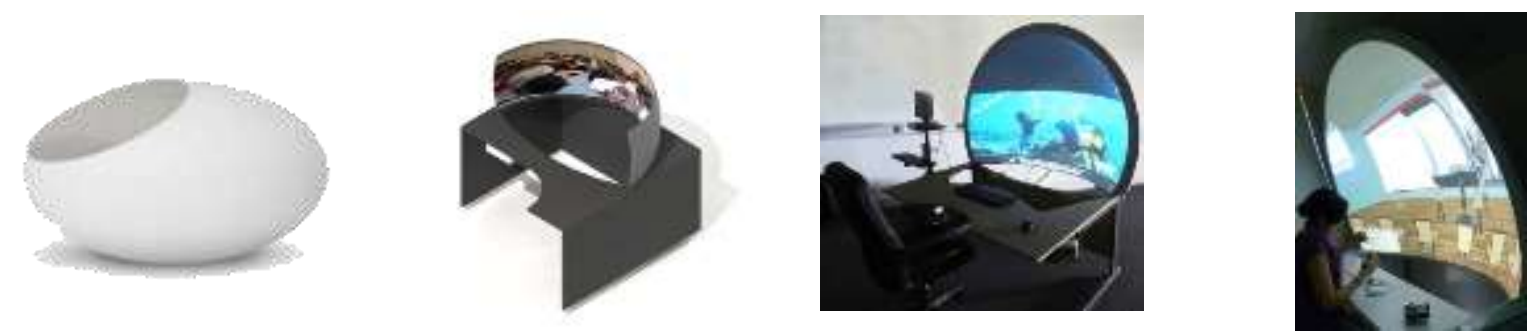

Figure 2: (from left to right) the original SFP egg-like concept, the eDesk concept (image courtesy of Immersive Displays Ltd), alpha prototype, beta-prototype

\footnotetext{
${ }^{2}$ http://victor.callaghan.info/publications/2010_CS10(TalesFromAPod).pdf
} 
This SFP might have remained fiction if it hadn't been for a meeting with David J Aldridge the CEO of a small Essex based SME, Immersive Displays Ltd $^{3}$, that produce virtual-reality environments such as flight simulators, planetariums and exhibition systems. Talking with them revealed they could build some aspects of the ePod now, albeit in a somewhat different form, as a student desk; the eDesk. The result was the product depicted in figures $2 \& 3$.

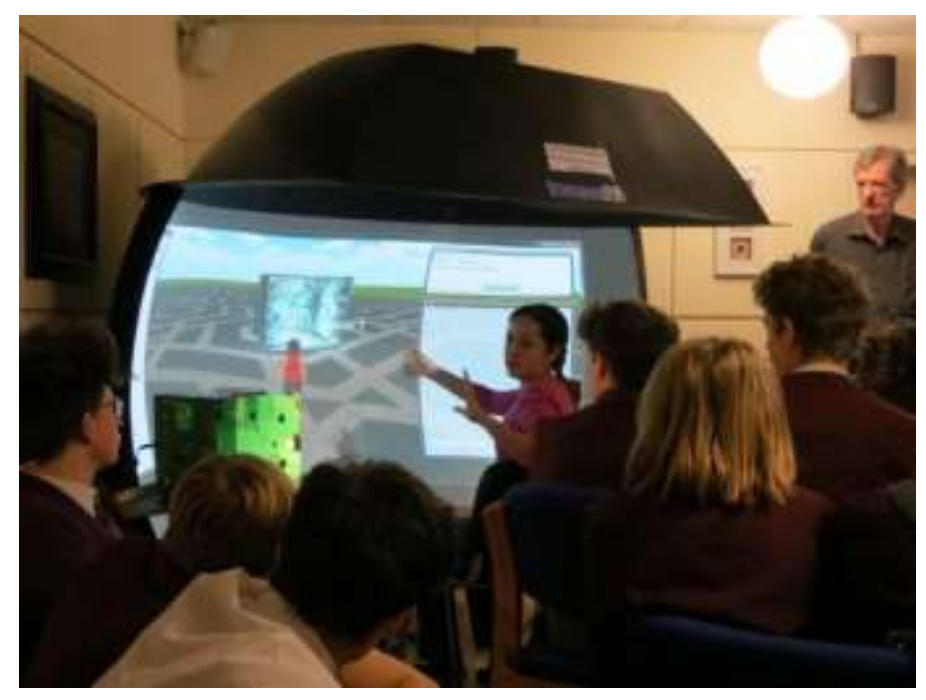

Figure 3: the final production model.

In the final eDesk product, the desk and spherical screen (ImmersaVU) were constructed by Immersive Displays $\mathrm{Ltd}^{2}$, the mixed-reality education-ware by Fortito $\mathrm{Ltd}^{4}$ (a small Mexican SME) with the mixed-reality, vision and Al software created by Anasol Pena-Rios, a PhD student at University Essex ${ }^{5}$ \& King Abdulaziz University ${ }^{6}$. The resultant system was tested successfully is a trial that interconnected students in the UK, China, Malaysia and Mexico into a unified mixed-reality laboratory session where the students collaborated to build smart home systems using a mix of hard and soft components which functioned as a collective whole despite the large geographical separations. A YouTube video shows this system in action, see http://youtu.be/ZjnnBCMKrmc.

\section{The $21^{\text {st }}$ Century Robot Project}

This is a crowd-sourced enterprise enabling society at large to participate in a Maker activity which aims to explore the possibilities for creating future domestic robots. The project is based around the skeleton of a humanoid robot which participants are invited to customise by adding their own 3Dprinted skins and software functionalities (via App modules).

The main participants in the $21^{\text {st }}$ Century Robot project ${ }^{7}$ are Intel $^{8}$ (makers of the Edison embeddedprocessor), Trossen Robotics ${ }^{9}$ (makers of the exoskeleton) and $\mathrm{O}^{\prime}$ Reilly ${ }^{10}$ (organisers of the

\footnotetext{
${ }^{3}$ http://www.immersivedisplay.co.uk/

4 http://fortito.mx/

${ }^{5}$ http://www.essex.ac.uk/

${ }^{6}$ http://www.kau.edu.sa/home_ENGLISH.aspx

7 http://www.21stcenturyrobot.com/

8 http://www.intel.com

9 http://www.trossenrobotics.com

10 http://www.oreilly.com/
} 
supporting Maker Fayres and publishers of the related book). The concept arose from a SFP series called, "Nebulous Mechanisms", written by Brian Johnson about a robot called Jimmy, and the issues that arose through mimicking the irrational aspects of humans in robots (one of the directions $\mathrm{Al}$ could explore as it moves towards the technological singularity).
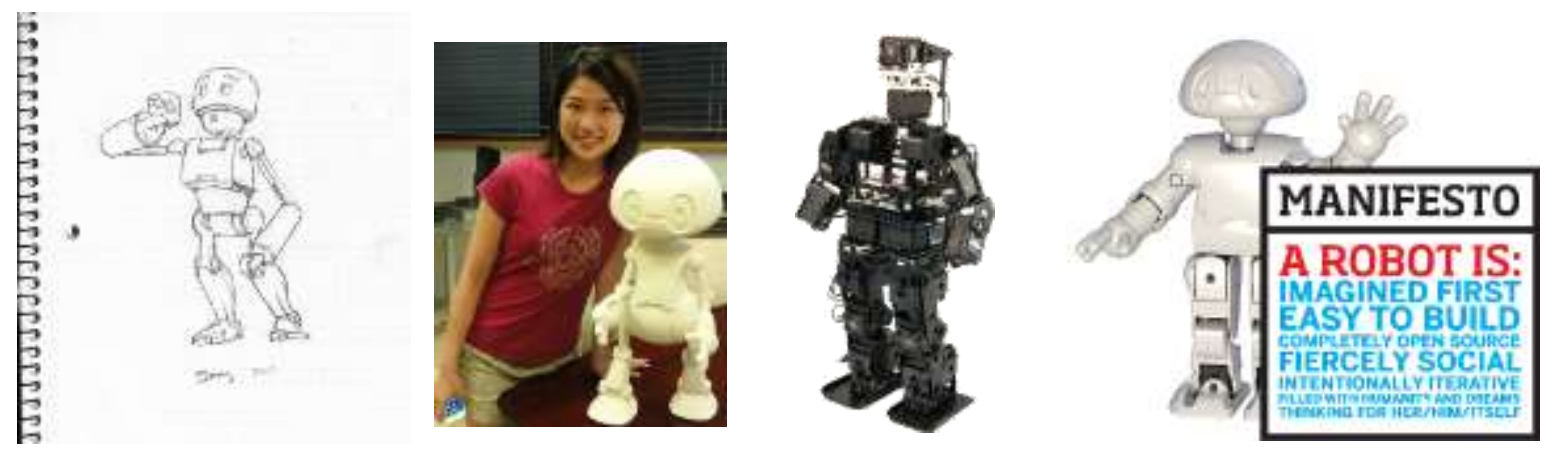

Figure 4: (from left to right) the original concept sketch, an early prototype (with Hsuan-Yi Wu), the production exoskeleton version (image courtesy of Trossen Robotics $\left.{ }^{9}\right) t$ and a version with a 'Jimmy skin' that mirrors the associated Maker book (image courtesy of Maker Media).

This project is exploring many issues of importance to $21^{\text {st }}$ Century industry such as the consequences of moving from a centralised to a more distributed mode of manufacturing plus the possibilities for rebalancing customer-company relationships through ideas such as SFP and open innovation.

\section{Other activities}

Beyond company based innovation, the Creative Science Foundation is working towards supporting the introduction of creative approaches to science, engineering and business education. Examples include a computer security course in Washington University (USA), entrepreneurship at Canterbury Christ Church University (UK), Imagination Workshops (Business Innovation) at the National Taiwan University (Taiwan), Computer English in Shijiazhuang University (China) and motivating preUniversity students towards STEM choices via schemes such as Essex University's (UK) 'New Creatives'. It's interesting to note that, in China, every university computer science student is required to undertake a 'Computer English' course. SFP is perfect for exercising language and in cooperation with Tsinghua University Press, the Creative Science Foundation is writing a standard textbook to integrate learning language, creative thinking and computer science, which will be available to all Chinese students; a potentially significant development for Chinese IT education.

\section{And finally ....}

Creative thinking is important for the success of any organisation and much has been written about this over the years. In this spirit the Creative Science Foundation seeks to pick up that baton for those of us who were inspired into IT careers by science fiction and the possibilities of applying the power of IT to transform our world for the better. For more information about CSf, or how to become involved, see www.creative-science.org or http://linkd.in/1xUMdJD. 